\title{
Microplane constitutive model and metal plasticity
}

\author{
Michele Brocca and Zdeněk P Bažant \\ Department of Civil Engineering and Materials Science, Northwestern University, Evanston IL; \\ z-bazant@nwu.edu
}

\begin{abstract}
The microplane model is a versatile constitutive model in which the stress-strain relations are defined in terms of vectors rather than tensors on planes of all possible orientations, called the microplanes, representative of the microstructure of the material. The microplane model with kinematic constraint has been successfully employed in the modeling of concrete, soils, ice, rocks, fiber composites and other quasibrittle materials. The microplane model provides a powerful and efficient numerical tool for the development and implementation of constitutive models for any kind of material. The paper presents a review of the background from which the microplane model stems, highlighting differences and similarities with other approaches. The basic structure of the microplane model is then presented, together with its extension to finite strain deformation. Three microplane models for metal plasticity are introduced and discussed. They are compared mutually and with the classical $J_{2}$-flow theory for incremental plasticity by means of two examples. The first is the material response to a nonproportional loading path given by uniaxial compression into the plastic region followed by shear (typical of buckling and bifurcation problems). This example is considered in order to show the capability of the microplane model to represent a vertex on the yield surface. The second example is the 'tube-squash' test of a highly ductile steel tube: a finite element computation is run using two microplane models and the $J_{2}$-flow theory. One of the microplane models appears to predict more accurately the final shape of the deformed tube, showing an improvement compared to the $J_{2}$-flow theory even when the material is not subjected to abrupt changes in the loading path direction. This review article includes 114 references.
\end{abstract}

\section{INTRODUCTION}

Over the past two decades, the microplane model has been successfully used by Bažant and coworkers to model the mechanical behavior of quasibrittle materials such as concrete, soils, rocks, ice, fiber composites, stiff foams and shape memory alloys [see eg, Bažant (1984), Bažant and Prat (1988a,b), Carol and Bažant (1997), Brocca et al (1999a,b)]. Several practical applications (eg, large-scale finite element analysis of concrete structures at WES) have shown the efficiency and accuracy of the model.

In a microplane model, the stress-strain relations are defined in terms of vectors rather than tensors independently on planes of many different orientations, called the microplanes, approximately representative of the microstructure of the material. The stress and strain components on a particular microplane are called the microplane stress and strain components. The overall macroscopic behavior, in terms of the usual macroscopic stress and strain tensors, is obtained by superimposing the effects of all the microplanes.

One appealing aspect of this approach is that it provides the researcher with an efficient theoretical and numerical framework, within which the constitutive law is simple. Once the general algorithm for dealing with the relationship between microplane quantities and macroscopic stress and strain tensors has been established, formulating a constitutive law is conceptually simple and intuitive, since all the quantities involved have always an immediate physical meaning.
In this article, we will discuss three different models for metal plasticity developed with the microplane approach. We will also present a theoretical comparison of the microplane model with the major existing phenomenological and crystallographic models of metal plasticity. Such a comparison is particularly meaningful in this case, because the original theoretical background from which the microplane model stems was historically developed in the field of metal plasticity for polycrystalline metals. We will review the approaches and models most widely used in this field and will try to clarify the differences and similarities with the microplane model. We will show in the following that the microplane model stands somewhere in between the two extremes represented by phenomenological and crystallographic models and can thus be considered a semi-phenomenological model.

Finally, we will compare quantitatively the microplane model and the classical $J_{2}$-flow theory, by considering two numerical examples: material response for a non-proportional loading path with a sudden change in direction and the tube-squash test on a ductile steel carried out at Northwestern University.

\section{REVIEW OF MAJOR EXISTING MODELS FOR METAL PLASTICITY}

Metal plasticity is usually modeled following two major approaches: a crystallographic one, which is based on micro- 
mechanical considerations and tries to reproduce closely the physical phenomena whose macroscopic manifestation is plastic deformation, and a phenomenological one, wherein plastic deformation is characterized in terms of the stress and strain tensors and their invariants. The latter approach is completely unrelated to the microscopic mechanisms of plastic deformation.

The crystallographic theories can be classified into two main groups: Taylor models and self-consistent models. Section 2.1 will review the Taylor models and briefly address the self-consistent models.

Another approach that draws inspiration from crystallographic experimental observation, is the slip theory of plasticity, proposed by Batdorf and Budiansky (1949) as an adaptation of the idea of Taylor(1938). This approach differs from the strictly crystallographic ones, because the material is modeled as a continuum, neglecting the crystalline structure and the anisotropy of the lattice. The slip theory of plasticity will be reviewed in Section 2.2. The microplane model is an adaptation and generalization of the slip theory of plasticity.

The most common phenomenological theory for elasticplastic deformation of metals is the Prandtl-Reuss flow theory with isotropic hardening, or $J_{2}$-flow theory. A modification of this theory, which allows more accurate predictions of material response in the case of sudden changes of loading path direction, is the $J_{2}$-comer theory of Christoffersen and Hutchinson (1979). The phenomenological theories and methods will be briefly discussed in Section 2.3 .

\subsection{Crystallographic models}

The models reviewed in this section assume that the microscopic source of plastic deformation is crystallographic slip. Such assumption is based on physical considerations. These models produce large strain constitutive laws that can be used to interpret experiments on large strain plastic behavior of metals. They also provide a basis for formulating phenomenological constitutive laws (eg, Christoffersen and Hutchinson (1979) used the results from Hutchinson's (1970) small-strain, rate-independent polycrystal calculations to specify corner characteristics in their $J_{2}$-corner theory).

The idea for the crystallographic approach to material modeling was inspired by experimental observations of crystallographic behavior of metals. Single crystal tests by Taylor and Elam $(1923,1925,1926)$ showed that under high stress, slip occurs on certain crystallographic planes along certain directions. They observed that slip on a given plane depends on the resolved shear stress on that plane and is independent of the normal stress on the plane. The stress at which slip occurs is called the critical stress. The increase of the critical stress with the magnitude of slip is known as strain hardening, while latent hardening is the increase in critical stress in unslipped systems.

Taylor (1934) showed in his dislocation theory that sliding occurs in such a manner that perfect crystal structure is reformed after each atomic jump. The lattice structure of the bulk of the material remains essentially the same after the occurrence of slip, hence the elastic modulus remains the same. The elastic strain is caused by the elastic deformation of the lattice structure, and the plastic strain is caused by the movement of dislocations. A discussion of the dislocation mechanism background of this continuum slip description of plastic flow is given by Asaro (1983a).

The Taylor-type models and self-consistent schemes explicitly represent the deformation by crystallographic slip in polycrystals. Polycrystals are continuous three-dimensional collections of grains (ie, crystallites), each of which can deform by the mechanism of crystallographic slip. Taylor (1938) proposed a model that strictly enforces compatibility by imposing the same set of strains (aggregate strains) on each grain. This idea comes from the experimental observation that most grains of a polycrystal undergo about the same strain.

Taylor (1938) used his model to analyze simple tension or compression of single phase FCC (face-centered cubic) polycrystals. In Taylor's numerical calculations, the polycrystal is made initially isotropic by choosing the grains to have a uniform coverage of all crystallographic orientations. The only source of inelastic deformation is assumed to be the crystallographic slip. The calculation is then based on determining the combination of slip systems and corresponding shear strains and stress state in each grain required to produce the specified strain. The selection of slip systems required to produce an arbitrary strain is not necessarily unique. Taylor made the physically intuitive assumption that among all the possible choices for combinations of active slip systems, the appropriate choice was that for which the cumulative shears are minimized. (actually that for which the net internal work is minimized, which reduces to minimization of cumulative shears if all the shear systems have same shear strength and hardening). Taylor could predict textures in FCC crystals in agreement with experiments of axisymmetric tension and compression.

Bishop and Hill (1951) proposed a polycrystal theory based on the principle of maximum work. They used inequalities between external work, computed as the product of macroscopic stress and strain increments, and internal work, computed as the integral over the volumes of grains of the products of the crystallographic shear strength and assumed slip increments, to set bounds on the critical stress state required to induce yield. Their primary interest was to determine yield surfaces.

The Taylor type models have been extensively used in the past 50 years to predict texture development and stress strain response. The development in this field is in the direction of increasing complexity, but most of the models retain the basic assumptions and structure of the Taylor model. We will now outline the common assumptions and the basic formulation of the Taylor model, as developed and used, among others, by Rice (1971), Hill and Rice (1972), Asaro and Rice (1977), Asaro (1983b), Asaro and Needleman(1985), Harren et al (1989), and Bronkhorst et al (1992).

The stress response at each macroscopic continuum material point is given by the volume-averaged response of the multitude of microscopic single crystalline grains comprising the material point. It is assumed that all the grains have equal volume, and that the local deformation gradient in each grain is homogeneous and identical to the macroscopic deforma- 
tion gradient $\boldsymbol{F}$ at the continuum material point. The mechanics of crystal deformation by slip is represented in two parts: the plastic deformation is given by a set of plastic simple shears from the reference configuration on the slip system of the crystallite, and the lattice with its embedded material elastically deforms and rigidly rotates from this plastically sheared state to reach the current configuration [Rice (1971), Asaro and Rice (1971)]. The deformation gradient tensor $F$ is therefore decomposed as

$$
\boldsymbol{F}=\boldsymbol{F}^{2} \boldsymbol{F}^{p}
$$

where tensor $F^{p}$ describes the material shear flow along the various slip systems of the crystallite, and tensor $F^{e}$ describes the elastic distortion of the lattice along with the rigid rotation of the crystallite.

The plastic shear flow from the reference configuration is written as

$$
L^{p}=\dot{F}^{p} F^{p-1}=\sum_{\alpha} \dot{\gamma}^{(\alpha)} s^{(\alpha)} m^{(\alpha)}
$$

where $\dot{\gamma}^{(\alpha)}$ is the shear rate on slip system $\alpha$. The slip system $\alpha$ is defined by the unit crystallographic vectors $s^{(\alpha)}$ and $m^{(\alpha)}$, where $s^{(\alpha)}$ is the direction of slip in the actual configuration and $m^{(\alpha)}$ is normal to the slip plane in the actual configuration. The plastic response of a crystallite is defined in terms of the resolved shear stress on each slip system. Each of the slip systems is active as long as the resolved shear stress on that system does not vanish. The plastic shear rate on the slip system $\alpha$ is taken to be governed by a constitutive function of the form

$$
\dot{\gamma}^{(\alpha)}=\dot{\gamma}^{(\alpha)}\left(\tau^{(\alpha)}, g^{(\alpha)}\right)
$$

where $\tau^{(\alpha)}$ is the resolved shear stress for the slip system $\alpha$, and $g^{(\alpha)}$ is the shear resistance of the slip system $\alpha$. This equation can be given in the form of a power law [Hutchinson (1965), Pan and Rice (1983), Pierce et al (1983)].

An evolution law can be given for $g^{(\alpha)}$ in the form

$$
\dot{g}^{(\alpha)}=\sum_{\beta} h_{\alpha \beta}\left|\dot{\gamma}^{(\beta)}\right|
$$

where $h_{\alpha \beta}$ is the rate of strain hardening on slip system $\alpha$ due to shearing on the slip system $\beta$ (latent hardening is thus taken into account).

The elastic response of the crystallite is governed by the elastic moduli set up to take into account the anisotropy due to crystallite orientation. In some cases, researchers consider the elastic response of polycrystalline aggregate, but neglect the elastic anisotropy of the FCC single crystal, and the elasticity tensor is the usual elasticity tensor.

Once the behavior of a crystallite is defined, the constitutive response of a polycrystalline aggregate is obtained using an averaging scheme [described in Hill (1972), and Asaro and Needleman (1985)].

The foregoing constitutive model can be used in two types of finite element calculations:

1) Those where the integration point represents a material point in a polycrystalline sample and the constitutive re- sponse is given through a Taylor-type polycrystal model; and

2) those where the integration point represents a material point in a single grain and the constitutive response is given through a single crystal model without invoking the Taylor assumptions.

In this second kind of computation both equilibrium and compatibility are satisfied in a weak finite element sense. In Taylor-type computations, the compatibility is satisfied, but not equilibrium between grains.

Bronkhorst et al (1992) presented an evaluation of the Taylor model, done by comparing finite-element results to experimental results. They concluded that the Taylor-type model is in a reasonable first order agreement with the observation of the texture formation and also with the overall stress-strain response of single-phase copper. Some deficiencies in the prediction from the Taylor model (finite element computations of the first kind) may be a consequence of the strong kinematic constraint on the deformation of the individual grains of the polycrystal in this model. In the finite element computations of the second kind, the constraint on the deformation of the individual grains is relaxed and the computation is run using initially random grain orientations. In this second case each element represents a single grain. When such an approach is adopted, a typical orange peel effect can be observed on the unconstrained surfaces of the FE mesh. This is caused by differences in the orientations of the grains that intersect the free surfaces, and is observed also in physical experiments.

To improve the performance of the model, the strong kinematic constraint characteristic of the Taylor model can be relaxed, by introducing intergranular constraint relations of various sorts. Butler and McDowell (1998) presented a reformulation of the kinematics of the plastic velocity gradient, introducing a new concept for taking into account the grain subdivision using additional plastic rotation associated with generation of the geometrically necessary dislocations. The modeling of grain subdivision is motivated by the recent experimental studies of microtexture formation [Hughes and Hansen (1993), Hughes (1995)]. The subdivision process facilitates adherence to the Taylor assumption of uniform deformation at the scale of individual grains. Butler and McDowell (1998) introduce a generalization of the decomposition of $F$

$$
F=F^{e} F^{\mu s} F^{p}
$$

which includes a part, $F^{4 s}$, that is associated with the subdivision, distinct from the contribution of dislocation glide, $F^{p}$. Here, $\boldsymbol{F}^{e}=\boldsymbol{R} \boldsymbol{U}^{e}$ includes the elastic stretch and, for small elastic strains, essentially rigid rotation relative to the lattice.

Another way to tackle the problem of excessive kinematic constraint in Taylor assumption is by enforcing the so-called relaxed constraints (RC) to reflect the inhomogeneity of strain from grain to grain that is expected to develop with increasing deformation. When grains become too distorted, enforcement of stress equilibrium rather than full compatibility may perhaps be more reasonable; this is taken as the basis of 
the RC model [Honneff and Mecking (1978), Canova, Kocks, Jonas (1984), Rollet et al (1989)].

Another approach to polycrystal modelling is the selfconsistent scheme suggested for this purpose by Kröner (1961), Budiansky and Wu (1962) and Hill (1965). This approach produces rate-independent models which attempt to account for grain interaction by considering each grain to be an inclusion embedded in an infinite homogeneous matrix whose moduli are the overall moduli of the crystal to be determined as an average over all grains. Hutchinson (1970) used this approach to determine the yield surfaces and stressstrain response for uniaxial tension and for uniaxial tension followed by shear (1970).

Brown (1970) and Berveiller and Zaoui (1979) extended the self-consistent models to account for rate dependence. In self-consistent models, the assumption of identical grain deformation is relaxed and these models also account, approximately, for intergranular equilibrium. They do so by enforcing equilibrium between the individual grains and the aggregate average. However, in self-consistent models as well as in Taylor type models, the deformation within each grain is presumed homogeneous.

\subsection{Slip theory of plasticity}

The slip theory, introduced by Batdorf and Budiansky (1949), assumes the plastic slip to be the only source of plastic deformation. The slip in any direction along parallel planes of any particular orientation in the material gives rise to a plastic shear strain, which depends only on the history of the corresponding component of shear stress. The plastic strain due to any system of applied stress is found by:

1) considering the history of the component in each direction of the shear stress on each plane of the material,

2) finding the corresponding plastic shear strain,

3) transforming this plastic shear strain into plastic strains in some fixed system of coordinates, and

4) summing over all the slip directions and slip plane orientations. In their original paper, Batdorf and Budiansky proposed a semigraphical method for the computations.

The shear stress required to produce slip is assumed to be independent of the normal stress and the amount of slip. The plastic shear deformation resulting from slip on a plane of a given orientation depends only upon the history of the component of the shear stress in the direction of slip on that plane.

Batdorf and Budiansky's approach differs substantially from the Taylor-type models and self-consistent models in that it neglects the actual crystallographic structure of the material and the anisotropy that it implies. The metal is modeled by considering it to be a macroscopically isotropic continuum, and slip is possible on a plane of any direction at any given point, and not only on the planes whose orientation is determined by crystallographic considerations. The theory contemplates an infinitesimal plastic shear strain associated with each infinitesimal fraction of the continuum comprising all possible planes. Given a plastic shear strain on a given plane, the contribution of this infinitesimal shear strain to the strains in the macroscopic reference system can be expressed by projecting the shear strain along the $x, y, z$ directions.
The initial critical shear stress for slip on a plane is given by $1 / 2$ of the elastic limit in pure tension or compression, since in tension test the maximum shear stress is $1 / 2$ of the applied uniaxial stress. Beyond this initial value, the critical stress increases according to a characteristic shear function to be determined experimentally, from tests on the polycrystalline continuum, not on the single crystal. The slip theory of plasticity can therefore be considered to be semiphenomenological, since the assumed mechanism of plastic deformation is suggested by crystallographic observation. But the mathematical description of slip is derived in a phenomenological way.

Batdorf and Budiansky showed that their slip theory yields more accurate predictions than the deformation theory of plasticity or the $J_{2}$-flow theory in the cases characterized by an abrupt change in the loading path. They considered tests on thin aluminum alloy cylinders, compressed into the plastic range and then twisted while the compressive strain is held constant.

\subsection{Phenomenological models}

The simplest and most commonly used phenomenological theory for infinitesimal elastic-plastic deformation of polycrystalline metals is the classical, rate-independent, PrandtlReuss flow theory with isotropic hardening based on the von Mises yield criterion [eg, Hill (1950)]. This classical rateindependent model has been generalized to finite deformation and a frame indifferent form by Hill $(1958,1959)$. The rate-independent plasticity has been studied, among others, by Hibbitt et al (1970), Needleman(1972), and Osias and Swedlow (1974).

The numerical implementation of the infinitesimal strain version of the rate-independent model into displacementbased finite element procedures dates back to the 1960 s (Wilkins, 1964), and the study of the numerical implementation of the finite-strain, frame indifferent version of the rateindependent and rate-dependent models started in the following decade [McMeeking and Rice (1975), Hughes (1984), Needleman (1984), Anand (1982, 1985), Brown et al (1989), etc]. These models usually employ the additive decomposition of the stretch tensor into elastic and plastic parts. For a numerical implementation of a model based on multiplicative decomposition of the deformation gradient into elastic and plastic parts, see Weber and Anand (1990).

These finite deformation constitutive models are in essence extensions of the small-strain isotropic hardening plasticity models, and therefore they are expected to provide accurate descriptions of the deformation behavior of initially isotropic materials only up to deformation levels where significant anisotropy in the metal has not yet developed.

The criterion for physical soundness of a plasticity theory (leaving aside damage mechanics) is assumed to be the satisfaction of Drucker's postulate. Drucker (1951) proposed a definition of work-hardening on the basis of some quasithermodynamic arguments. This led to the following two inequalities:

$$
\dot{\sigma}_{i j} \dot{\varepsilon}_{i j}^{p}>0
$$




$$
\left(\sigma_{i j}-\sigma_{i j}^{*}\right) \dot{\varepsilon}_{i j}^{p} \geq 0
$$

where $\sigma_{i j}$ is the current stress state on the yield surface $S$ and $\sigma_{i j}^{*}$ is any other stress state in $E$ (elastic region). The second inequality is called the principle of maximum plastic work by Bishop and Hill(1951) who prove it for single crystals that deform plastically by slip. Inequality (2.7) has the following two consequences:

1) the yield surface is convex;

2) If the current stress state $\sigma_{i j}$ is on $S$ and $S$ is smooth at $\sigma_{i j}$, then, for any given loading $\dot{\sigma}_{i j}$, the direction of the strainrate vector $\dot{\varepsilon}_{i j}^{\mu}$ must be that of the exterior normal to the yield surface at $\sigma_{i j}$.

If $S$ is not smooth at $\sigma_{i j}$ (there is no unique normal at $\sigma_{i j}$ to surface elements of $S$ ), then $\dot{\varepsilon}_{i j}^{\mu}$ must merely point toward the cone of normals to $S$ at $\sigma_{i j}$.

It is well known that the classical theory of the PrandtlReuss flow rule (with an isotropically hardening smooth von Mises yield surface), ie, the $J_{2}$-flow theory, yields results in disagreement with the prediction of crystallographic slip models. The crystallographic slip models predict the existence of corners (or vertices) on the yield surface and thus a dependence of the plastic strain rate on the direction of the rate of stress. This is called the vertex effect. In the $J_{2}$-flow theory, on the other hand, the plastic strain rate is always normal to the von Mises yield surface.

The simplest vertex model for plasticity is the hypoelastic deformation theory of Stören and Rice (1975). In this theory, the plastic strain rate is not necessarily normal to what would be the von Mises yield surface. Being a deformation theory, it is not directly applicable to plastic loading paths that exhibit significant deviation from proportionality.

The $J_{2}$-comer theory of Christoffersen and Hutchinson (1979) eliminates this restriction. In this theory, the yield surface vertex is modeled as a stress space hypercone. For plastic loading along paths that coincide or nearly coincide with proportional loading, the response is taken as that of hypoelastic deformation theory. This regime of behavior is called the total loading. Elastic unloading occurs when the direction of the stress rate lies in or within the cone surface. For loading paths that lie between total loading and elastic unloading, the Christoffersen-Hutchinson theory provides a region of transitional response where the instantaneous moduli smoothly increase from the deformation theory moduli of the total loading regime to the linear elastic moduli of the unloading regime. The $J_{2}$-corner theory has been extended by Hutchinson and Tvergaard (1980) to include hyperelastic total loading response. A discussion about methods for endowing the general incremental theory of plasticity with a vertex was also given in Bažant $(1980,1987)$.

The high curvature of the yield surface in the neighborhood of its current loading point can be described to some extent by the classical theory of kinematic hardening. Contributions in this sense have been given, among others, by Lee et al (1983), Dienes (1979), Key (1984), Fressengeas and Molinari (1985). Harren et al (1989) compare the poly- crystal predictions to predictions based on phenomenological vertex-type descriptions. They do so by performing large shear calculations with $J_{2}$-corner theory and two versions of kimematic hardening theory. For plane strain incompressible deformation, the $J_{2}$-comer theory and the FressengeasMolinari kinematic hardening theory agree well with polycrystal predictions.

A different phenomenological representation, which more closely reflects the physical origin of the vertex effect, was given by Sewell (1974), who used the theory of multiple yield systems, introduced by Koiter (1953), Mandel (1965) and Hill (1966). The formulation for multisurface plasticity develops from the assumption that, at each state, the plastic strain consists of several components,

$$
d \varepsilon_{i j}^{p}=\sum_{i} d \varepsilon_{i j}^{p(i)}
$$

each of them governed by a different yield surface. This formulation yields in principle a rather realistic description of material behavior, but it is not easy to apply because identification of the material parameters from test data is difficult [see also eg, Bažant and Cedolin (1991)].

In the 1970s, another approach has received considerable attention: the endochronic theory [eg, Valanis (1971), Bažant $(1978,1980)]$. The basic concept in the endochronic formulation is the characterization of inelastic strains in terms of one (or several) non-decreasing scalar variables whose increments depend on the strain increments. This variable is generally called the intrinsic time although it doesn't necessarily (and normally) correspond to physical time. Similar to vertex hardening models and the deformation theory of plasticity, the endochronic theory gives inelastic strain for strain increments that are tangential to the current loading surface. However, in contrast to vertex hardening, the endochronic inelastic strain for tangential strain increments is normal to the loading surface. Consequently, the endochronic theory is stiffer than vertex hardening for this loading direction. Although the endochronic approach is very convenient for describing hysteresis, it is purely phenomenological, with $\mathrm{nr}$ clear physical basis, and seems to have lost popularity.

The $J_{2}$-flow theory will serve in Section 6 as a reference for the numerical evaluation of the microplane models for plasticity. The most common ways of implementing numerically the $J_{2}$-flow theory are finite strain extensions of the algorithm developed by Wilkins in 1964 . Two algorithms have been used for comparisons with the microplane model: one presented by Hughes (1984) and the other one by Ponthot (1995). They are based on two different ways of approximating the incremental strain at each time step. Both approaches employ the radial return algorithm [presented by Wilkins (1964) and generalized by Krieg and Key (1976)]. In the examples considered here, the two algorithms yield nearly identical results.

\section{REVIEW OF MICROPLANE MODEL}

The microplane model developed by Bažant and coworkers is an evolution and generalization of Batdorf and Budiansky's approach It was first used to model concrete, rocks, and soils. 
Carol and Bažant (1997) pioneered the microplane models for plasticity. In this paper, three new models for plasticity within the microplane model framework are formulated.

\subsection{History of microplane model}

The background history of the microplane model can be traced back to the pioneering idea of GI Taylor (1938), presented in Section 2.1. As seen in Section 2.2, Batdorf and Budiansky (1949) extended Taylor's idea and developed a realistic model for plasticity of metals, still considered among the best. Many other researchers subsequently refined or modified this approach to metals [Kröner (1961), Budiansky and Wu (1962), Lin and Ito (1965, 1966), Hill (1965, 1966)]. Extensions for the hardening inelastic response of soils and rocks where also made [Zienkiewicz and Pande (1977), Pande and Sharma (1981, 1982)].

The slip theory of plasticity used the so called static constraint, that is the assumption that the stress vector acting on a given plane in the material, called the microplane, is the projection of the macroscopic stress tensor. Later Bažant (1984) showed that the static constraint induces unstable localizations of softening into a plane of one orientation, which makes it very difficult to generalize the model for post-peak strain-softening damage of quasibrittle materials. The extension to strain-softening damage calls for replacing the static constraint by a kinematic constraint, in which the strain vector on any inclined plane in the material is the projection of the macroscopic strain tensor.

The expression slip theory of plasticity is unsuitable for general material models, for example models of the cracking damage in quasibrittle materials, where the inelastic behavior on the microscale does not physically represent slip. For this reason the neutral term microplane model was coined, applicable to any type of inelastic behavior [Bažant (1984)]. Microplane is the name given to a plane of any orientation in the material, used to approximately characterize the physical phenomena occurring in the microstructure of the material on planes of that orientation.

After generalizing the microplane model for both tensile and compressive damage [Bažant and Prat (1988a,b), Bažant et al (1996)], the microplane model and the corresponding numerical algorithm reached its present, very effective formulation for concrete in Bažant et al (2000a,b,c) and Caner et Bažant (2000). Microplane formulations have also been developed for anisotropic clays [Bažant and Prat (1987)] and for soils [Prat and Bažant $(1989,1991 \mathrm{a}, \mathrm{b})]$. A detailed review of the microplane model formulation with kinematic or static constraint can be found, eg, in Carol and Bažant (1997). For both the formulations with kinematic constraint and with static constraint, the material properties are characterized by relations between the components of the stress and strain vectors on the microplanes. The tensorial invariance restrictions need not be directly enforced in the constitutive relations, which is a simplifying feature of the microplane formulation. They are automatically satisfied by superimposing in a suitable manner the responses from the microplanes of all orientations. This is done by means of a varia- tional principle (principle of virtual work), as introduced in Bažant (1984).

The next paragraphs will present the basic formulation for the microplane model for the case of small strains. Generalization to the finite strain range will be discussed in Section 4.

\subsection{Formulation with kinematic constraint}

The orientation of a microplane is characterized by the unit normal $\mathbf{n}$ of components $n_{i}$ (indices $i$ and $j$ refer to the components in Cartesian coordinates $x_{i}$ ). In the formulation with kinematic constraint, which makes it possible to describe softening in a stable manner, the strain vector $\vec{\varepsilon}_{N}$ on the microplane (Fig 3.1) is the projection of the macroscopic strain tensor $\varepsilon_{i j}$. So the components of this vector are $\varepsilon_{N i}=\varepsilon_{i j} n_{i}$. The normal strain on the microplane is $\varepsilon_{N}=n_{i} \varepsilon_{N i}$, that is

$$
\varepsilon_{N}=N_{i j} \varepsilon_{i j} ; \quad N_{i j}=n_{i} n_{j}
$$

where repeated indices imply summation over $i=1,2,3$. The mean normal strain, called the volumetric strain $\varepsilon_{V}$, and the deviatoric strain $\varepsilon_{D}$ on the microplane can also be introduced, being defined (for small strains) as follows:

$$
\varepsilon_{V}=\varepsilon_{k k} ; \quad \varepsilon_{D}=\varepsilon_{N}-\frac{1}{3} \varepsilon_{V}=\frac{2}{3}\left(\varepsilon_{N}-\varepsilon_{S}\right)
$$

where $\varepsilon_{S}=$ spreading strain $=$ mean normal strain in the microplane. $\varepsilon_{S}$ characterizes the lateral confinement of the microplane and governs the creation of splitting cracks normal to the microplane. Considering $\varepsilon_{V}$ and $\varepsilon_{D}$ (or $\varepsilon_{S}$ ) is useful when dealing with the effect of lateral confinement on compression failure and when the volumetric-deviatoric interaction, typical of cohesive frictional materials such as concrete, needs to be captured.

To characterize the shear strains on the microplane (Fig 3.1 ), we need to define two coordinate directions $M$ and $L$, given by two orthogonal unit coordinate vectors $m$ and 1 of components $m_{i}$ and $l_{i}$ lying on the microplane. To minimize the directional bias of $\mathbf{m}$ and $\mathbf{l}$ among the microplanes, one may alternate among choosing vectors $m$ to be normal to axis $x_{1}, x_{2}$, or $x_{3}$.

The magnitudes of the shear strain components on the microplane in the directions of $\mathrm{m}$ and $\mathrm{l}$ are $\varepsilon_{M}=m_{i}\left(\varepsilon_{i j} n_{i}\right)$ and $\varepsilon_{L}$ $=l_{i}\left(\varepsilon_{i j} n_{i}\right)$. Because of the symmetry of tensor $\varepsilon_{i j}$, the shear strain components may be written as follows [eg, Bažant et al $(1996,2000 \mathrm{c})]$ :

$$
\varepsilon_{M}=M_{i j} \varepsilon_{i j} ; \quad \varepsilon_{L}=L_{i j} \varepsilon_{i j}
$$

in which the following symmetric tensors are introduced:

$$
M_{i j}=\left(m_{i} n_{j}+m_{j} n_{i}\right) / 2 ; \quad L_{i j}=\left(l_{i} n_{j}+l_{j} n_{i}\right) / 2
$$

Once the strain components on each microplane are obtained, the stress components are updated through microplane constitutive laws, which can be expressed in an algebraic or differential form.

If the kinematic constraint is imposed, the stress components on the microplanes are equal to the projections of the macroscopic stress tensor $\sigma_{i j}$ only in some particular cases, when the microplane constitutive laws are specifically prescribed so that this condition be satisfied. This happens for example in the case of elastic laws at the microplane level, 
defined with elastic constants chosen so that the overall macroscopic behavior is the usual elastic behavior [see Carol and Bažant (1997)]. In general, the stress components determined independently on the various planes will not be related to one another in such a manner that they can be considered as the projections of a macroscopic stress tensor.

Thus static equivalence or equilibrium between the microlevel stress components and macrolevel stress tensor must be enforced by other means, in a weak sense. This can be accomplished by applying the principle of virtual work, which yields

$$
\sigma_{i j}=\frac{3}{2 \pi} \int_{\Omega} \sigma_{N} n_{i} n_{j} \mathrm{~d} \Omega+\frac{3}{2 \pi} \int_{\Omega} \frac{\sigma_{T r}}{2}\left(n_{i} \delta_{r j}+n_{j} \delta_{r i}\right) \mathrm{d} \Omega
$$

where $\Omega$ is the surface of a unit hemisphere. Equation (3.5) is based on the equality of the virtual work inside a unit sphere and on its surface, rigorously justified by Bažant et al (1996).

The integration in Equation (3.5), is performed numerically by an optimal Gaussian integration formula for a spherical surface using a finite number of integration points on the surface of the hemisphere. Such an integration technique corresponds to considering a finite number of microplanes, one for each integration point. A classical formula of adequate accuracy for microplane applications, consisting of 28 integration points, is given by Stroud(1971). Bažant and Oh (1986) developed a more efficient and about equally accurate formula with 21 integration points, and studied the accuracy of various formulas in different situations.

\subsection{Formulation with static constraint}

A formulation with static constraint equates the stress components on each microplane to the projections of the macroscopic stress tensor $\sigma_{i j}$. Once the strain components on each microplane are updated by the use of the microplane constitutive laws, the macroscopic strain tensor is obtained again by applying the principle of virtual work.

The microplane components of stress are obtained as follows:

$$
\begin{array}{ll}
\sigma_{N}=N_{i j} \sigma_{i j} ; & N_{i j}=n_{i} n_{j} \\
\sigma_{M}=M_{i j} \sigma_{i j} ; & \sigma_{L}=L_{i j} \sigma_{i j}
\end{array}
$$

where

$$
M_{i j}=\left(m_{i} n_{j}+m_{j} n_{i}\right) / 2 ; \quad L_{i j}=\left(l_{i} n_{j}+l_{j} n_{i}\right) / 2
$$

The complementary virtual work equation provides, in analogy to equation (3.5),

$$
\varepsilon_{i j}=\frac{3}{2 \pi} \int_{\Omega} \varepsilon_{N} n_{i} n_{j} \mathrm{~d} \Omega+\frac{3}{2 \pi} \int_{\Omega} \frac{\varepsilon_{T r}}{2}\left(n_{i} \delta_{r j}+n_{j} \delta_{n i}\right) \mathrm{d} \Omega
$$

Again, volumetric and deviatoric quantities can be introduced:

$$
\sigma_{V}=\sigma_{k k} / 3 ; \quad \sigma_{D}=\sigma_{N}-\sigma_{V}
$$

$\sigma_{V}$ and $\sigma_{D}$ are used when the effect of hydrostatic pressure and spreading stress or confining stress need to be accounted for explicitly.

\subsection{Formulation with double constraint}

It is possible and advantageous to formulate the microplane model with particular material laws such that a kinematic constraint for the strains coexists with a static constraint for the true stresses in the sense of damage mechanics (but of course not with the actual stresses). When this happens the model is said to have a double constraint since it satisfies simultaneously the integral equations (3.5) for strains and (3.9) for true stresses. Such a double constraint is useful in microplane damage formulations [Carol and Bažant (1997), Bažant et al (1996, 2000)]

Figure 3.2 shows schematically the pattern followed in order to update stress or strain in the load steps for an explicit algorithm for the microplane model. As seen from Fig 3.2 , the microplane model takes a simple constitutive law on each microplane and transforms it into a consistent threedimensional model.

When the kinematic constraint is used, the macroscale strain tensor is projected onto the microplanes using Eqs (3.1)-(3.4). Microplane constitutive laws (described in Section 5) are applied on each microplane, producing the stresses on each of the microplanes. The macroscopic stress is then determined numerically via integration of Eq (3.5).

When the static constraint is used, the macroscale stress tensor is projected onto the microplanes using Eqs (3.6)(3.8). Stresses on each of the microplanes are obtained applying microplane constitutive laws.

Finally the macroscopic stress is computed numerically via integration of $\mathrm{Eq}$ (3.9). The numerical procedure is incremental, and small increments of stress are taken at each step.

\section{GENERALIZATION OF MICROPLANE MODEL TO FINITE STRAINS}

A systematic and detailed discussion about how to extend the microplane model to very large strains (of the order of $100 \%$ ) is given by Bažant et al (1998). Here we present only

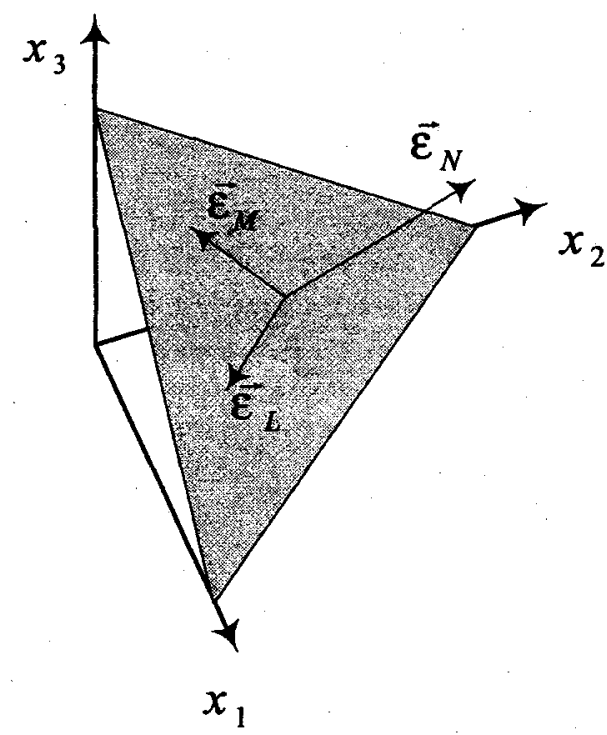

Fig 3.1. Strain components on a microplane 
a brief review of the main concepts on which such an extension in based.

Let us consider a broad class of strain measures called the Doyle-Ericksen tensors [Doyle and Ericksen (1956), Ogden (1984), Bažant and Cedolin (1991)]:

for $m \neq 0: \quad \varepsilon^{(m)}=\frac{1}{m}\left(U^{m}-I\right)$,

for $m=0: \quad \varepsilon^{(m)}=\ln U$

where $U=\sqrt{C}, C=F^{T} F$, and $m$ is a parameter which could be any real number. $C$ is the Cauchy-Green deformation tensor; $U$ is called the right-stretch tensor and is defined by the polar decomposition $\boldsymbol{F}=\boldsymbol{R} \boldsymbol{U}$ of the deformation gradient $\boldsymbol{F}=$ $\partial x / \partial X$, where $x$ and $X$ are, as usual, the final and initial Cartesian coordinates of the material point [Ogden(1984), Bažant and Cedolin (1991), Malvern (1969)]. (The tensor products such as $\boldsymbol{R} U$ are singly contracted products, ie, the dot symbol for product is omitted.)

For $m=2$, (4.1) yields the Green's Lagrangian strain [Malvern (1969)]:

$$
\varepsilon=\frac{1}{2}\left(F^{T} F-I\right)
$$

For $m=1,(4.1)$ yields the Biot strain tensor, and for $m \rightarrow 0$ the Hencky (logarithmic) strain tensor [Hencky (1928), Ogden (1984), Bažant and Cedolin (1991), Rice (1993)]. Formulations corrsponding to $m=-1$ and $m=-2$ are also found in the literature. The stress tensor $S$ for which $S$ :de is the correct work expression (called the conjugated stress tensor) is the second Piola-Kirchhoff stress tensor, which is related to the Cauchy (true) stress tensor $\sigma$ by the following relations:

$$
S=F^{-1} J \sigma F^{-T} \text { or } \sigma=J^{-1} F S F^{T}
$$

where $F^{-T}=\left(F^{-1}\right)^{T}=\left(F^{T}\right)^{-1}$ [Ogden(1984), Bažant and Cedolin (1991), Malvern (1969)].

The decomposition of large deformations into their volumetric and deviatoric parts is, in general, multiplicative. It has the form $U=F_{D} U_{V}$ [Flory (1961), Sidoroff (1974), Simo and Ortiz (1985), Bell (1985), Lubliner (1986), Simo (1988)] where $U=$ right stretch tensor, $U_{V}=$ volumetric right stretch tensor, and $\boldsymbol{F}_{D}=$ deviatoric transformation tensor. For con- crete and many other materials, the volumetric-deviatoric decomposition is simplified by the fact that the volume changes are always small. In that case, the decomposition can approximately be written as additive [Bažant (1996)]. In component form it reads $\varepsilon_{i j}=\varepsilon_{D i j}+\varepsilon_{\nu} \delta_{i j}$, where $\varepsilon_{V}$ is the exact expression for the volumetric strain for the given strain measure. For Green's Lagrangian strain measure, $\varepsilon_{V}=\varepsilon_{0}+$ $1 / 2 \varepsilon_{0}^{L}$, with $\varepsilon_{0}=(J-1) / 3(J=\operatorname{det} F=$ Jacobian of the transformation) and the additive approximation is acceptable up to a volume change of $3 \%$. For Biot strain measure $\varepsilon_{V}=J^{1 / 3}$ -1 , and the approximation is acceptable up to a volume change of $8 \%$. The additive decomposition is exact if and only if the strain measure is the Hencky (logarithmic) strain tensor $\boldsymbol{H}$, in which case $\varepsilon_{V}=(\ln J) / 3$. For concrete, the volume change is $-3 \%$ at the highest pressures tested so far (2069 MPa; Bažant, Bishop and Chang, 1986). Thus the classical multiplicative decomposition, which is less practical for calculation than the additive decomposition, seems to be inevitable only for materials exhibiting very large volume changes, such as solid foams.

Bažant and coauthors (2000a) show that for an efficient formulation of a microplane constitutive model with physical. meaning, the best choice of strain tensor is the Green's Lagrangian strain tensor, while the best choice of stress tensor is the back-rotated Cauchy (true) stress tensor. Although these strain and stress tensors are nonconjugate, they can still be admissible because the following four conditions are satisfied: 1) there is a unique correspondence between the nonconjugate constitutive law and the conjugate constitutive law in terms of Green's Lagrangian strain tensor; 2) if a micromacro kinematic constraint of microplane model is imposed in terms of one type of strain tensor, a kinematic constraint is satisfied for any other finite strain tensor; 3) the elastic parts of strains are always small, which ensures the energy dissipation caused by elastic deformation formulated in terms of nonconjugate stress and strain tensors to be always negligible; and 4) in the time steps of the numerical algorithm, the inelastic stress drops to the yield or boundary surface occur at constant strain and always dissipate energy. For a detailed discussion of these four conditions see Bažant et al (2000a). In the following we will consider the motivations that lead to the choice of the back-rotated Cauchy stress and of the Green's Lagrangian strain tensor.

\section{Choice of stress tensor for microplane model:}

In order to exploit fully the advantage of formulating a material constitutive model in the microplane approach, it is necessary that the stress components on the microplanes characterize the true stress on planes of various orientations within the material. If this condition is satisfied it is possible to use
Microplane Model with kinematic constraint
Fig 3.2. Patterns for stress or strain update in the microplane models with static or kinematic constraint

Standard approach to material modeling

Microplane Model with static constraint

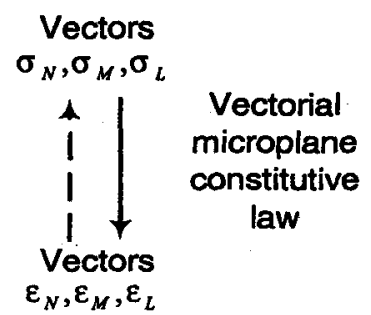


directly the normal and shear components on the microplanes to express inelastic phenomena such as friction and strength or yield limit.

It can be shown that for large stretches such as 2 or $1 / 2$, the compoments of the second Piola-Kirchhoff stress tensor can enormously differ from the corresponding components of the Cauchy stress tensor, easily by a factor of 2 or more or even in sign. Consequently, the limit condition of frictional slip on a certain plane cannot be formulated in terms of $S$ components on that plane, and neither can the limit conditions of yield or strength limit, or the law of strain hardening on that plane. Frictional slip on a certain plane, for example, depends on the true normal and shear stresses on a section by that plane through the deformed material, and not directly on stresses transformed to a section of the material in its virgin initial state. Similar conclusions can be reached for other easily calculated stress tensors conjugated to other finite strain tensors, such as Biot stress tensor. Another relevant observation is that a constitutive law in terms of the second Piola-Kirchhoff stress does not allow simple control of hydrostatic pressure, and thus also of pressure sensitivity, internal friction and dilatancy of material. Further examples of difficulties with the use of $S$ to express the yield limit in shear and the internal friction angle have been given by Bažant (1997). Again, similar difficulties can be demonstrated for stresses conjugate to other finite strain tensors, eg, Biot's.

The Cauchy stress tensor would give a clear physical meaning for the microplane stress components, but it cannot be used in a constitutive equation for a solid with memory of the initial state because it is not referred to the initial configuration and is not conjugated by work with any Lagrangian finite strain tensor. It is conjugate to the deformation rate (or velocity strain) used in Eulerian formulations.

The proper stress tensor to use is the Cauchy stress tensor rotated back to the initial coordinates $X$ attached to the material, called the back-rotated Cauchy stress tensor and expressed as

$$
s=R^{T} \sigma R
$$

where $\sigma$ is the Cauchy stress tensor, and $R$ is the material rotation tensor. In the case of no rotation, $s$ coincides with the Cauchy stress tensor. If there is rotation $R$, the physical meaning of stresses does not get changed by it, only the component values get transformed. The hydrostatic pressure, which is important for pressure sensitive materials such as concrete, is given by tr $(s)$.

Another possible choice may be the back-rotated Kirchhoff stress tensor (not considered here), used by Hoger (1987), Eterovic and Bathe (1990) and Gabriel and Bathe (1995). It is also used in ABAQUS [Hibbitt et al (1995)] and EPIC [Johnson et al (1995)].

A constitutive relation written in terms of the back-rotated Cauchy stress or back-rotated Kirchhoff stress can, of course, be always transformed to a constitutive relation in terms of the second Piola-Kirchhoff stress. But after this transformation, the constitutive law becomes much more complicated and the microplane laws for different microplanes cease to be independent.

\section{Choice of strain tensor for microplane model:}

The first natural choice for the strain measure. would be the strain tensor that is work conjugate to the chosen stress tensor. In the case of microplane model, this is not possible for two reasons [Bažant et al (2000a)].

The first reason is that the strain tensors conjugate to theback rotated Cauchy stress and back-rotated Kirchhoff stress are both nonholonomic. In other words, they depend not only on the current deformation gradient, but also on the deformation path in which the current state has been reached. A path-dependent strain tensor appears questionable for materials for which the initial virgin state is an important reference for defining the constitutive behavior, such as fracturing damage in concrete.

The second reason is that, in a finite strain generalization of the microplane model, a definite physical meaning needs to be attached to the normal and shear strain components on the microplanes. The following two conditions must be met:

Condition $I$. The normal strain component $\varepsilon_{N}$ on a microplane should uniquely characterize the stretch $\lambda_{N}$ of a material line segment whose direction $\boldsymbol{n}$ is initially normal to the microplane, and it should be independent of the stretches of material line segments in other initial directions.

Condition II. The shear strain component $\varepsilon_{N M}$ (or $\varepsilon_{N L}$ ) should uniquely characterize the change of angle $\theta_{N M}$ or $\theta_{N L}$ between two initially orthogonal material line segments with initial unit vectors $n$ and $\boldsymbol{m}$. It should be independent of the stretch in any direction and of the angle change in any plane not parallel to $n$.

Only the Green's Lagrangian strain meets these two conditions [Bažant et al (2000a)].

\section{MICROPLANE MODELS FOR METAL PLASTICITY}

The microplane model presented in Sections 3 and 4 offers an efficient theoretical and numerical framework for implementing material models. We will now present three microplane models for metal plasticity, substantially different from each other:

1) a microplane model version of the $J_{2}$-flow theory;

2) a microplane model version of the slip theory of plasticity (which uses a static constraint);

3) a microplane model for plasticity based on independent yield conditions for shear and deviatoric components of strain(with kinematic constraint).

\subsection{Microplane model version of $\mathbf{J}_{\mathbf{2}}$-flow theory (MP1)}

In this model (from now on referred to as MP1), the yield condition is given by a $J_{2}$-type function on the microplane level:

$$
f(\sigma)=\sigma_{D}^{2}+\sigma_{L}^{2}+\sigma_{M}^{2}-k^{2}=0
$$

When this condition is met, yielding occurs and radial return is performed on the stress components $\sigma_{D}, \sigma_{L}$ and $\sigma_{M}$. It can be shown that condition (5.1) is equivalent to the von Mises condition in the case of saturation, ie, states in which all the microplanes undergo yielding at the same time [Carol and Bažant (1997)]. To prove this, we recall that the second 
invariant of the deviatoric stress $\left(J_{2}\right)$ can be computed in terms of the microplane components of stress as

$$
2 J_{2}=\frac{3}{2 \pi} \int_{\Omega}\left(\sigma_{D}^{2}+\sigma_{L}^{2}+\sigma_{M}^{2}\right) d \Omega
$$

where $\Omega$ is the surface of a unit hemisphere, as in (3.5) or (3.9) [see Carol and Bažant (1997)]. For a saturated state of yielding, all the microplanes satisfy the condition

$$
\sigma_{D}^{2}+\sigma_{L}^{2}+\sigma_{M}^{2}=k^{2}
$$

This can be introduced in (5.2), which can then be integrated, yielding

$$
2 J_{2}=\frac{3}{2 \pi} \int_{\Omega}\left(k^{2}\right) d \Omega=\frac{3}{2 \pi} k^{2} \cdot 2 \pi=3 k^{2}
$$

Thus, a saturated state of yielding is characterized by the usual von Mises condition

$$
J_{2}=\frac{3}{2} k^{2}=\tau^{2}
$$

After yielding saturation, the performance of this model is very similar to that of the usual models of $J_{2}$-flow theory, such as those presented in Section 2.4. The major difference is that different planes, with different physical orientations come into play at different times, giving a smooth transition from elastic response to plastic response.

Figure 5.1 shows the uniaxial stress-strain curves for the same material obtained with a $J_{2}$-flow plastic law and with model MP1. With a $J_{2}$-flow plastic law, the material response changes from plastic to elastic suddenly. With the microplane model this transition occurs gradually, as the yield condition is met successively in different planes.

Model MPI cannot reproduce a vertex in the yield surface and deviation from normality. Because of the way in which the yield surface is defined on each microplane (indeed equivalent to a macroscopic $J_{2}$-flow model), when the increment in the macroscopic deviatoric stress is normal to the actual deviatoric stress, at the microplane level the increment in the stress components will be tangential to the yield surface. Therefore the initial shear stiffness predicted by this model in the case of shear following uniaxial pre-loading is the elastic shear stiffness, as it is when a $J_{2}$-flow theory model is used.

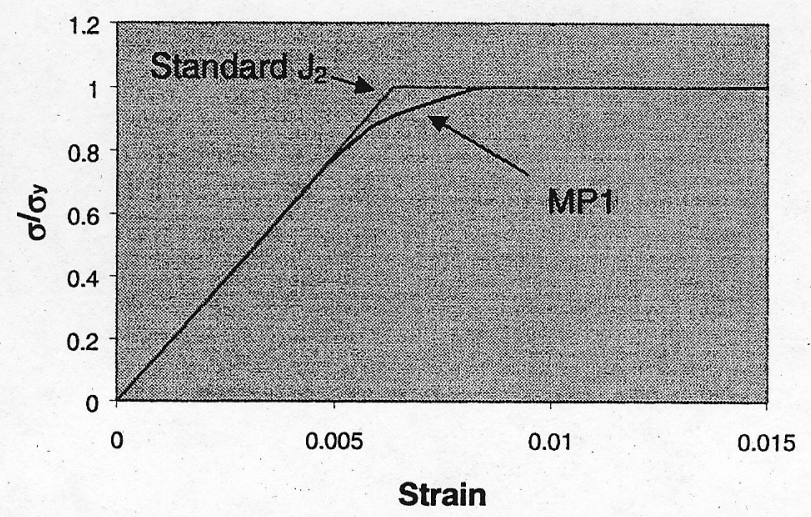

Fig 5.1. Uniaxial stress-strain curves for an elastic-perfectly plastic material as reproduced by model MP1 and a standard $J_{2}$-flow model.
A version of MP1 was originally introduced by Carol and Bažant (1997) who proposed an algorithm employing a yield condition rewritten in terms of microplane strains instead of stresses. However it turns out that employing condition (5.1), in terms of microplane stresses, yields more accurate results in the finite strain range.

\subsection{Microplane model version of slip theory of plasticity (MP2)}

In this model, the shear slip at the microplane level is assumed to be the only source of plastic deformation. As usual, the strain tensor is given by the summation of an elastic part and a plastic part:

$$
\varepsilon_{i j}=\varepsilon_{i j}^{e}+\varepsilon_{i j}^{p}
$$

The elastic part of the strain tensor is related to the stress tensor by the usual elasticity relationships. Yielding occurs when the shear component of stress at the microplane level exceeds a given value. The yield condition, therefore, is

$$
\sigma_{T}=\sigma_{y}\left(\bar{\varepsilon}^{p}\right)
$$

where $\sigma_{y}$ is a parameter determined experimentally, as a function of the effective plastic strain $\left(\bar{\varepsilon}^{p}\right.$, simply defined so that

$$
\left.d \bar{\varepsilon}^{p}=\sqrt{\left(d \varepsilon_{L}^{p}\right)^{2}+\left(d \varepsilon_{M}^{p}\right)^{2}}\right),
$$

and $\sigma_{T}^{2}=\sigma_{L}^{2}+\sigma_{M}^{2}$. When yielding occurs, slip produces a plastic shear strain at the microplane level, $\varepsilon_{T r}^{p}(r=1,2)$, and the plastic strain tensor $\varepsilon_{i j}^{r}$ is then obtained by superposition of the effect of slip on all the planes. Such a superposition is computed (in a weak sense) by using a formula analogous to (3.10):

$$
\varepsilon_{i j}^{p}=\frac{3}{2 \pi} \int_{\Omega} \frac{\varepsilon_{T r}^{p}}{2}\left(n_{i} \delta_{r j}+n_{j} \delta_{r i}\right) \mathrm{d} \Omega
$$

derived from the principle of complementary virtual work. This approach requires adopting the static constraint. When slip is the only source of plastic deformation, the distribution of plastic strain among the various planes is very nonuniform. The kinematic constraint would give a more uniform distribution of plastic slip strains among the microplanes, and thus the corresponding material response would inevitably be too stiff.

This model with static constraint is very similar to the model proposed by Batdorf and Budiansky (1949), called the slip theory of plasticity. The difference is in the integration scheme and the way the overall plastic strain tensor is computed.

Model MP2 can represent a vertex on the yield surface. The consequence of such a vertex is, for example, a reduction of shear stiffness in the case of a loading path in which shear follows uniaxial pre-loading into the yielding region. In this regard, see the numerical examples in Section 6.1.

A finite element computation with an explicit dynamic formulation requires a subroutine that updates the stress for a given strain. Model MP2, which computes the strain tensor for a given stress tensor, can still be used, provided that the microplane stress values in each step are obtained by iteration. 
Obviously this increases the computational time and makes MP2 less convenient than MP3, which is introduced next.

A statically constrained model is inherently less stable than a kinematically constrained model, because in a statically constrained model the strain distribution over the microplanes can localize into one or several microplanes a spurious way. This problem arises in the case of microplane constitutive laws which imply strain softening or elastic-perfectly plastic behavior. But spurious localization can sometimes occur also with mild hardening, when geometric nonlinearities are taken into account (in a manner similar to that demostrated by Rudnicky and Rice, 1974). For this reason it is not easy to extend MP2 to the finite strain range efficiently.

\subsection{Microplane model for plasticity} with kinematic constraint (MP3)

A formulation with kinematic constraint is necessary if we want to develop a microplane model for plasticity able to represent a vertex in the yield surface and at the same time efficient for finite strain explicit finite element analysis. As mentioned, a kinematic constraint is not suitable to deal with a model in which plastic slip is the only source of plastic deformation. To achieve satisfactory performance, it is possible to assume that the contribution to the overall plastic deformation is given not only by the shear components of strain on each microplane, but also by the deviatoric part of the normal component of strain on each microplane. This assumption is at this time driven by convenience and its physical justification is still unclear. But it is acceptable in a model that does not aim at a crystallographic description of material response.

Independent yielding conditions can be assumed for the shear stresses and the deviatoric part of the normal stresses at the microplane level. Yielding occurs when either one of the following conditions is met, independently:

$$
\begin{aligned}
& f_{a}(\sigma)=\sigma_{L}^{2}+\sigma_{M}^{2}-k_{a}^{2}=0 \\
& f_{b}(\sigma)=\sigma_{D}^{2}-k_{b}^{2}=0
\end{aligned}
$$

where the two hardening parameters $k_{a}$ and $k_{b}$ are determined experimentally and have to be specified independently.

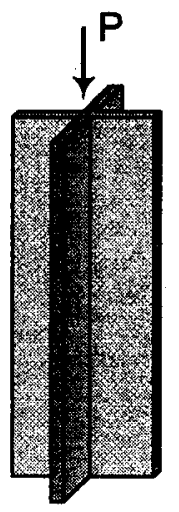

Fig 6.1. Cruciform column subjected to axial load

\section{NUMERICAL COMPARISONS}

\subsection{Non-proportional loading paths}

An important test for a plasticity model is to verify its capability of reproducing the apparent violation of normality in the case of loading paths characterized by abrupt deviations from proportionality. Deviation from normality is crucial for bifurcation phenomena and buckling problems [see for example Hutchinson (1974), or Bažant and Cedolin (1991)]. Experimentally this can be for instance revealed by uniaxial loading of a thin tube into the plastic region followed by torsion. Famous experiments evidencing deviations from normality were performed by Gerard and Becker (1957), who studied stability of stocky cruciform columns under axial load (Fig 6.1). The material used in Gerard and Becker's tests was aluminum 2024-T4.

Buckling of a cruciform column under compression was studied originally by Stowell $(1948 \mathrm{a}, \mathrm{b})$. If the column is not too slender, it undergoes torsional buckling, which involves


Fig 6.2. Variation of tangential shear modulus with axial stress 
twisting about its axis. In the elastic range, the compressive bifurcation load is

$$
P_{c r}=G J A / 2 I
$$

where $J=4 b h^{3} / 3=$ stiffness of the cross section in simple torsion, $I=2 b h^{3} / 3, h$ is the wall thickness, $b$ is the width of the flange plates, $A$ is the cross sectional area, and $G$ is the elastic shear modulus. The corresponding compressive stress is

$$
\sigma_{c r}=G(h / b)^{2}
$$

[see also Bažant and Cedolin (1991)]. In the plastic range, the first bifurcation load and stress are obtained by replacing $G$ with the tangent shear modulus $G_{t}$ in (6.1-2). $J_{2}$-flow plasticity yields for this case $G_{t}=G$ because the incremental deviatoric stress is tangent to the yield surface and thus the response is predicted as elastic. This means that the bifurcation load should be unaffected by plasticity. The results by Gerard and Becker clearly show that the bifurcation load is indeed affected by the applied compressive stress (and so is the tangential shear modulus) if the column is loaded into the plastic region.

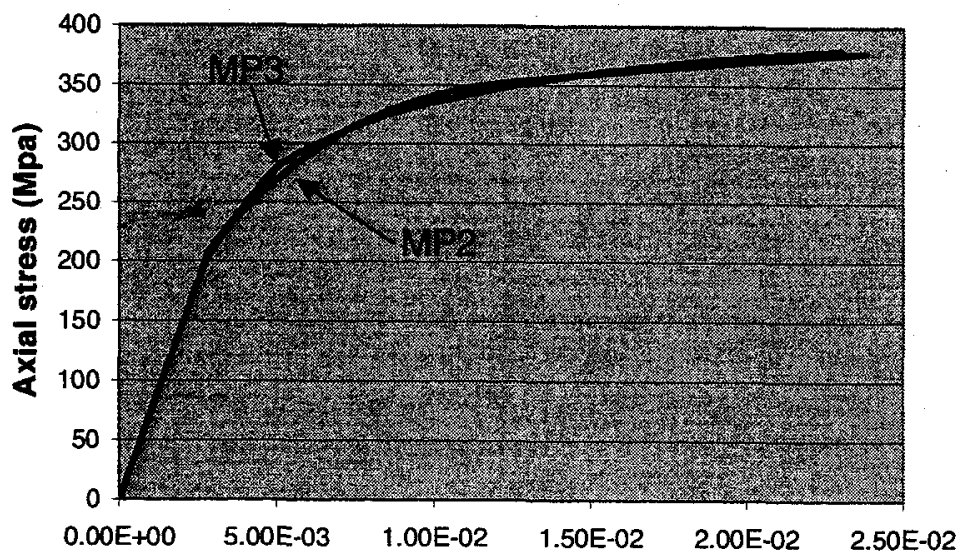

Axial strain

Fig 6.3. Assumed stress-strain curve for 2024-T4, in models MP2 and MP3

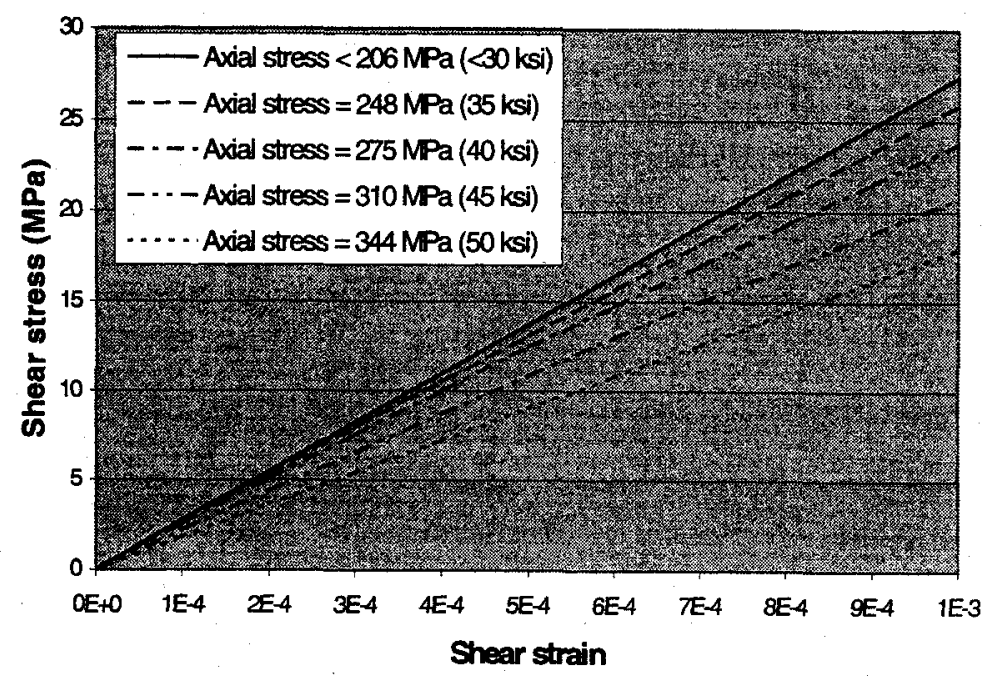

Fig 6.4. Predicted shear response after uniaxial pre-loading (model MP3)
The three microplane models presented in Section 5 have been used to fit the results of Gerard and Becker. The following material properties have been assumed for 2024-T4:

$$
\begin{array}{ll}
\text { Young's modulus } & \mathrm{E}=10.6 \times 10^{6} \mathrm{psi} \\
\text { Poisson's ratio } & v=1 / 3 \\
\text { Yield Stress (at } 0.2 \% \text { strain) } & \sigma_{y}=40 \mathrm{ksi}
\end{array}
$$

Figure 6.2a shows a comparison of the variation of $G_{t} / G$ predicted by the models MP1 and MP2 with the experimental results. As expected, model MP1 (equivalent to $J_{2}$-flow plasticity) predicts no reduction in the tangential shear modulus with increasing uniaxial stress. On the other hand, model MP2 can fit the experimental results up to the maximum applied compressive stress very accurately. Once the material parameters (6.3) are assumed, the hardening function for $\sigma_{y}\left(\bar{\varepsilon}^{p}\right)$ in (5.7) is determined so as to fit the experimental data in Fig 6.2a.

The corresponding uniaxial stress-strain curve is shown in Fig 6.3.

Figure $6.2 \mathrm{~b}$ shows the results obtained with model MP3. This time, the hardening functions $k_{a}, k_{b}$ in (5.9) are not adjusted by fitting Gerard and Becker's results. Rather they are chosen so that the corresponding uniaxial stressstrain curve reproduces closely that obtained with MP2 (Fig 6.3). With such hardening functions, model MP3 fits accurately the behavior observed experimentally up to $\sigma_{1}=327.5 \mathrm{MPa}$. After that, for increasing values of pre-applied uniaxial stress, the tangential shear modulus tends asymptotically toward a limit minimum value. Note that, since models MP2 and MP3 are different, obtaining very similar results in Fig 6.2a and Fig $6.2 \mathrm{~b}$, just by fitting the uniaxial stress-strain response, is a good evidence of their mutual consistency.

The fact that $G_{t} / G$ tends asymptotically to a limit value for MP3 is not surprising. The reason is that MP3 is a kinematically constrained model. In a statically constrained model (such as MP2), when the yield condition is met on some microplanes, the plastic deformation on such microplanes can reach large values (having no kinematic constraint). The strain can localize into a limited number of planes and the overall response of the material can be very compliant. On the other hand, when the kinematic constraint is imposed, the plastic deformation on the microplanes at which the yield condition is met is constrained by a limit posed by the deformation on the microplanes that respond elastically. When the applied load changes non-proportionally, as in the foregoing example, some of the microplane components of stress will keep loading into the plastic region, and other components will unload into the elastic region. It can be proven that the limit minimum value of $G_{d} / G$ following uniaxial loading is 0.5 for MP3. Such a value is obtained in the case in which, 
before the shear load is applied, the material is in a saturated plastic state (in which yielding is occurring for all the components of stress on all the microplanes), and the microplane constitutive laws are assumed to be elastic-perfectly plastic.

One further observation should be made looking at Fig $6.2 \mathrm{a}, \mathrm{b}$ : the response curve obtained with MP2 is wavy, while that obtained with MP3 is smoother. This is a manifestation of the fact that the statically constrained model is less stable than the kinematically constrained one and is thus prone to exhibit discontinuities in the response when more microplanes come into play.

To further illustrate the deviation from normality, Fig 6.4 shows the predicted shear response after uniaxial pre-loading for model MP3. The material parameters adopted for this example are the same as those used for the study on 2024-T4 aluminum (Fig 6.3). The results shown in Fig 6.4 are obtained in the following way: the material is first loaded in uniaxial compression up to a certain value of stress; then, in a second stage of loading, the material is loaded in shear while the uniaxial stress is kept constant. Some curves of shear stress versus shear strain are plotted for different values of the pre-applied uniaxial stress. The slope of these curves gives the tangential shear modulus. All these curves would be the same and equal to the curve with the highest slope if the $J_{2}$-flow model were used. Again it can be clearly observed that for increasing pre-loading the tangential shear stiffness is progressively reduced.

\subsection{Tube-squash test}

An interesting comparison of the performance of the $J_{2}$-flow theory and the microplane model can be made by considering the capability of these models to fit the experimental results of the 'tube squash' test (Fig 6.5) in a finite element simulation. This test has recently been developed (Bažant et $a l, 1999)$ in order to subject the material filling the tube, eg, concrete, to very large strain at very large pressures (shear angles up to $70^{\circ}$ have been achieved for concrete, without any cracking visually detectable on a cut). During the test, a thick tube made of a highly ductile steel alloy is squashed to one half of its original length. The particular steel alloy used in this test is capable of very large deformation without cracking. During the loading process the specimen bulges in the middle, thus deforming in a very non-homogeneous manner. For comparison, an empty steel tube must also be tested,

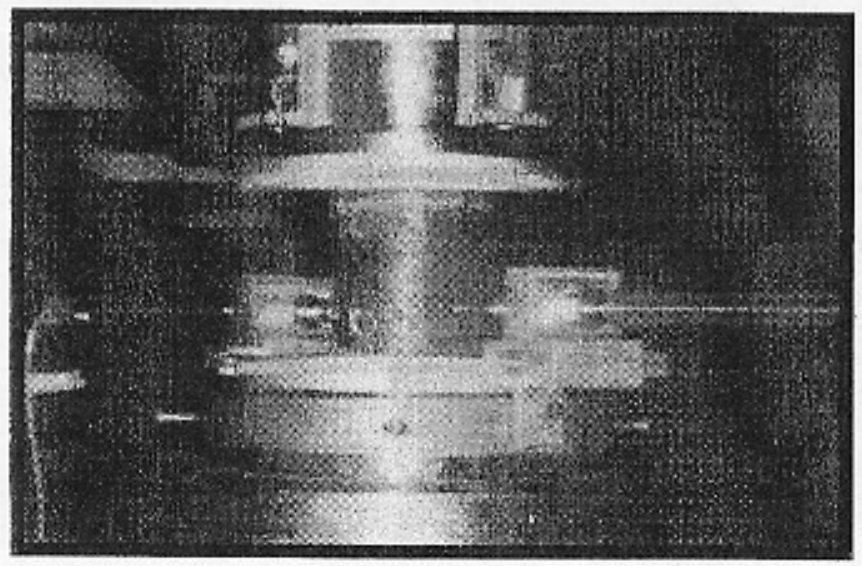

and this test is considered here. Figure 6.6 shows the photographs taken during the test

The finite element analysis is performed in a standard way. The problem is two-dimensional, due to axial symmetry of the geometry. An explicit dynamic finite strain finite element program has been written, within an updated Lagrangian framework. The time integration is carried out using central differences and the quasi-static nature of the loading process is reproduced by employing dynamic relaxation. For simplicity, perfect contact is assumed between the specimen and the loading plattens. This appears to be an acceptable simplification since no significant slip was observed in the test at the edge contact point between the specimen and the loading plattens.

Three finite element computations are run - with model
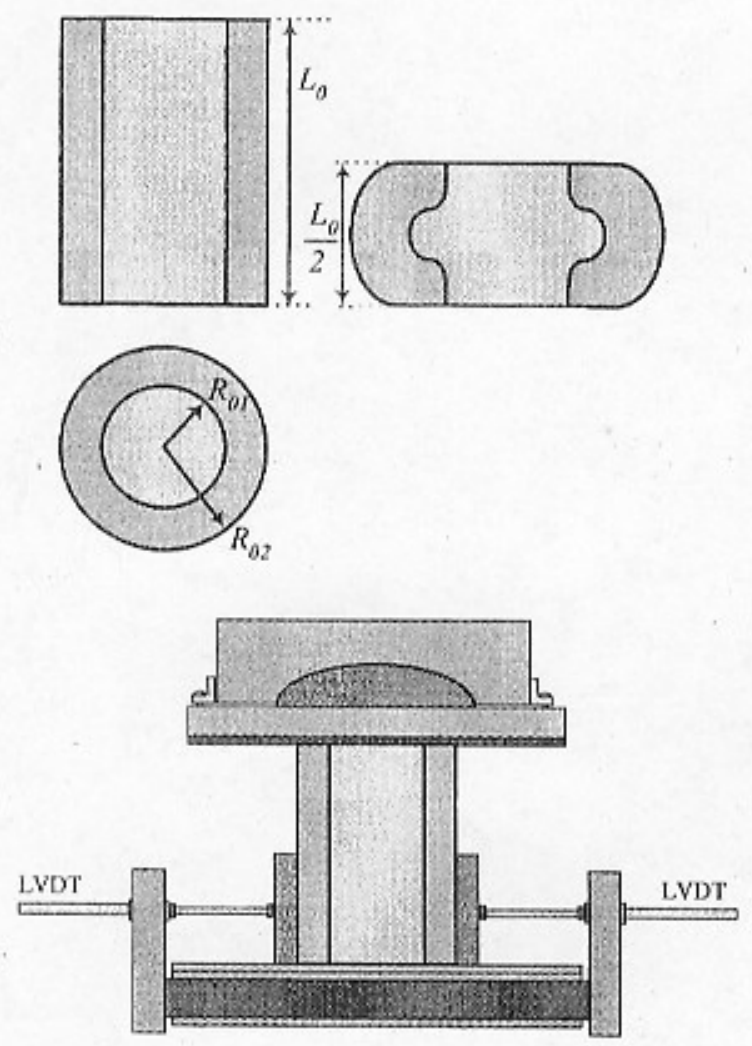

Fig 6.5. The specimen for the squash test.



Fig 6.6. The tube-squash test (after Bažant et al, 1999). 
MP1, model MP3, and the finite element code using the two $J_{2}$-flow algorithms mentioned in Section 2.4 (only the results relative to the first one of these two algorithms will be shown here, since the two algorithms yield in this case nearly identical results). The material parameters and the hardening functions in all the three models can be calibrated so that the measured load-displacement curve can be fitted very accurately: Figure 6.7 shows the load-displacement curve obtained

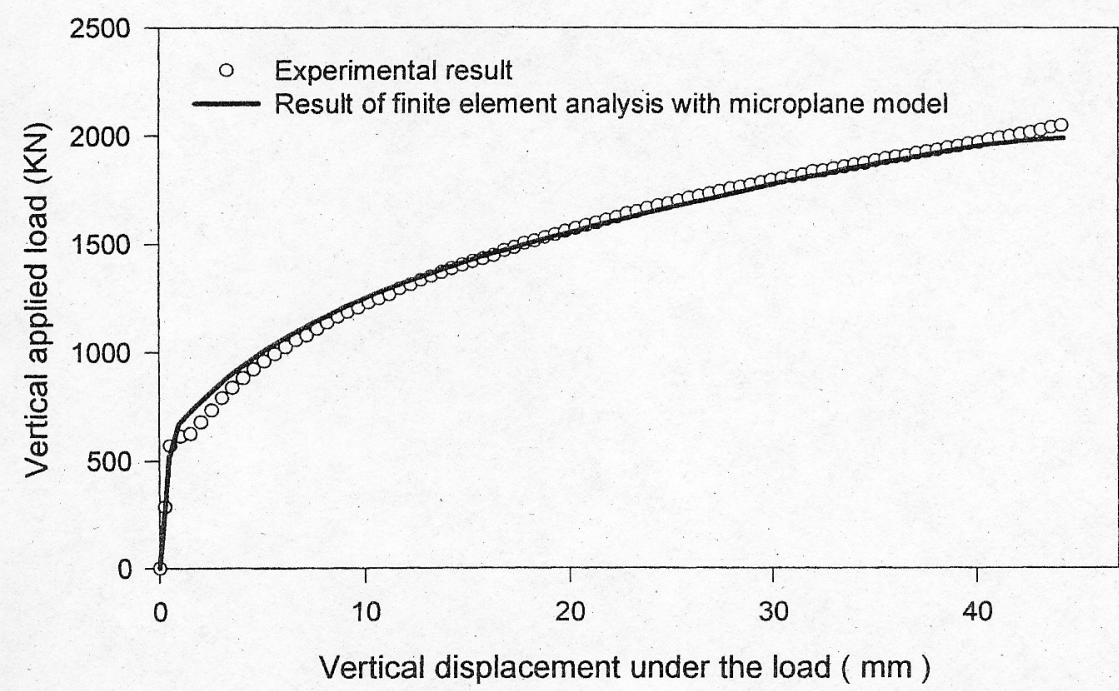

Fig 6.7. Experimental and computed load-displacement diagrams for the empty tube. with model MP3, compared to the experimental one. Similar fitting is easily obtained also for MP1 and for the computation with $J_{2}$-flow plasticity. However, the microplane models (and in particular model MP3) appear to be capable of a more accurate prediction of the final deformed shape of the tube.

Figure 6.8 shows the initial mesh and deformed mesh at the end of the loading process, as predicted by the standard $J_{2}$ flow plasticity (Fig 6.8b), using model MP1 (Fig 6.8c) and model MP3 (Fig 6.8d). The most accurate prediction among these three models is obtained with model MP3. A minor improvement with respect to the $J_{2}$-flow model can be observed also in the result obtained with model MP1. Such a model is based on a $J_{2}$-flow formulation at the microplane level, but it is implemented by assuming a different definition of stress and strain tensors as discussed in Section 4.

Note that the deviation from normality, which is crucial in the case of bifurcation studies, still appears to affect somehow the material behavior even in the case of the squash test, where the material in the bulge is subjected to a highly non-proportional loading path due to the significant rotations. This is doubtless the reason why model MP3 yields the best result.

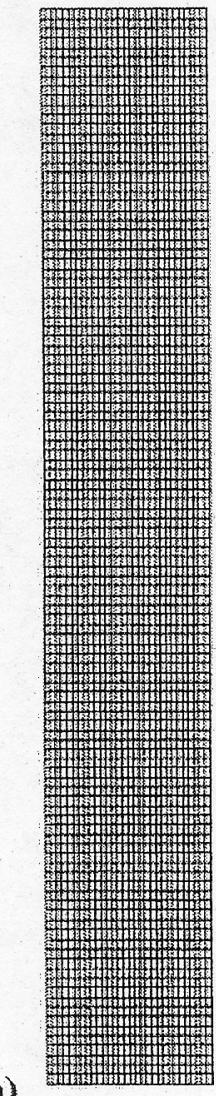

a)

b)

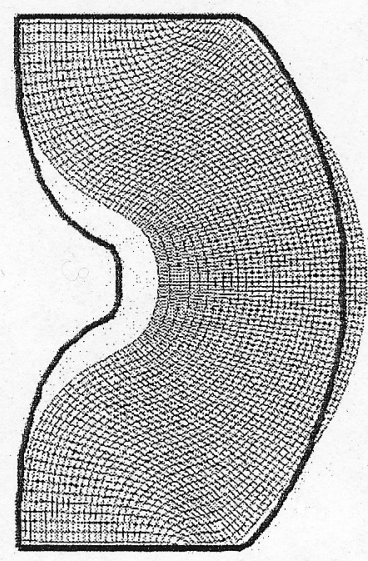

c)

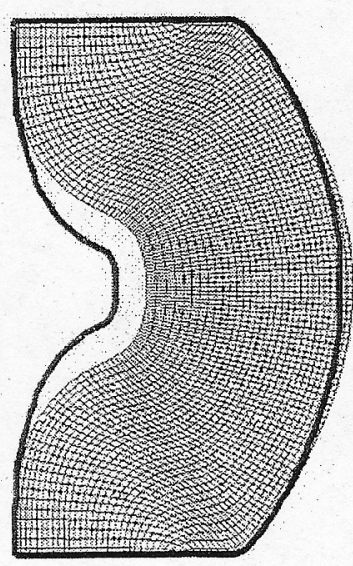

d)



Fig 6.8. Mesh used for the computations (a), and deformed profiles as predicted at the end of the loading process with J2 (b), MP1 (c) and MP3 (d). Three-dimensional visualization of the deformed mesh obtained with MP2 (e). 


\section{SUMMARY AND CONCLUSIONS}

The theoretical and numerical framework of the microplane model is very versatile. It provides a researcher with a powerful and efficient tool for the development and implementation of constitutive models for any kind of material. Three microplane models for metal plasticity have been introduced and discussed. The three models differ in the way the yielding condition is specified, and in the kind of constraint (kinematic or static) relating the microplane quantities to the macroscopic stress or strain tensors. The three models are different conceptually, but can all be easily implemented by simply specifying the constitutive law at the microplane level.

The microplane models have been compared quantitatively with the classical $J_{2}$-flow theory for incremental plasticity. Two cases have been considered. The first case is the material response to a nonproportional loading path given by uniaxial compression into the plastic region followed by shear (typical of buckling and bifurcation problems). This example is considered in order to show the capability of the microplane model to represent a vertex on the yield surface. The second case is the 'squash-test' of a highly ductile steel tube. Finite element computations have been run using two of the newly introduced microplane models and the $J_{2}$-flow theory. Both the microplane version of $J_{2}$-flow plasticity (model MP1) and the microplane model MP3 appear to predict more accurately the final shape of the deformed tube. The best result among the models considered in this particular example is obtained with model MP3. Model MP3 is also capable of reproducing a vertex on the yield surface at the loading point. This example shows that an improvement with respect to $J_{2}$-flow theory is attained even in a case in which the material is not subject to abrupt changes in the loading path direction.

\section{REFERENCES}

Anand L (1982), Constitutive equations for the rate-dependent deformation of metals at elevated temperatures. J Eng Mat Tech 104, 12.

Anand L (1985), Constitutive equations for hot-working of metals. Int $J$ Plasticity, 1, 213.

Asaro RJ (1983a), Micromechanics of crystals and polycrystals. Adv Appl Mech 23,1.

Asaro RJ (1983b), Crystal plasticity. J Appl Mech Trans ASME 50, 921-934.

Asaro RJ and Needleman A (1985), Texture development and strain hardening in rate dependent polycrystals. Acta Metall 33, 923-953.

Asaro RJ and Rice JR (1977), Strain localization in ductile single crystals. $J$ Mech Phys Solids, 33, 309-338.

Batdorf SB and Budiansky B (1949), A mathematical theory of slip based on the concept of slip. NACA TN1871, April.

Bažant ZP (1978), Endochronic inelasticity and incremental plasticity. Int $J$ Solids Struct, 14, 691-714.

Bažant ZP (1980), Work inequalities for plastic fracturing materials. Int $J$ Solids Struct, 16, 873-901.

Bažant ZP (1984), Microplane model for strain controlled inelastic behavior. Chapter 3 in Mechanics of Engineering Materials, CS Desai and RH Gallagher (eds), J Wiley, London, 45-59.

Bažant ZP (1987), Lectures on 'Material Modeling Principles' Course 720 D30, Northwestern Univ, Evanston IL.

Bažant ZP (1996), Finite strain generalization of small strain constitutive relations for any finite strain tensor and additive volumetric-deviatoric split. Int J Solids Struct 33 (20-22), 2887-2897 (special issue in memory of Juan Simo).

Bažant ZP (1998), Easy to compute finite strain tensors with symmetric inverse, approximating Hencky strain tensor. J Eng Mat Tech Trans ASME $120(2), 131-136$

Bažant ZP (1997), Recent advances in brittle-plastic compression failure: Damage localization, Scaling and finite strain. Computational plasticity:
Fundamentals and applications (Proc of $5^{\text {th }}$ Int Conf on Comput Plasticity, COMPLAS 5, held in Barcelona), DRJ Owen, E Onate and E Hinton (eds), publ by Int Center for Numer Meth in Eng, Barcelona, 3-19.

Bažant ZP, Adley MD, Carol I, Jirásek M, Akers SA, Rohani B, Cargile JD, and Caner FC (2000a), Large-strain generalization of microplane model for concrete and applications. J Eng Mech ASCE 126(9) 971-980.

Bažant ZP, Bishop FC, and Chang T-P (1986), Confined compression tests of cement paste and concrete up to $330 \mathrm{ksi}$. ACI Mat J, 33 (JulyAugust), 553-560.

Bažant ZP, Caner FC, Adley MD, and Akers SA (2000b), Incorporation of rate effects of fracturing and creep into microplane model M4 for concrete dynamics. J Eng Mech ASCE 126(9) 962-970.

Bažant ZP, Caner FC, Carol I, Adley MD, and Akers SA (2000c), Microplane model M4 for concrete: I. Formulation with work-conjugate deviatoric stress. J Eng Mech ASCE 126(9) 944-953.

Bažant ZP and Cedolin L (1991), Stability of structures: Elastic, inelastic, fracture and damage theories, Oxford Univ Press, New York.

Bažant ZP and Gambarova P (1984), Crack shear in concrete: Crack band microplane model. J Struct Eng, ASCE, 110, 2015-2035.

Bažant ZP and Kim J-K (1986), Creep of anisotropic clay: microplane model. $J$ Geotech Eng, ASCE, 112, 458-475.

Bažant ZP, Kim JJ and Brocca M (1999), Finite strain tube-squash test for concrete at high pressures and shear angles up to $70^{\circ} . A C I$ Mat $J, 96(5)$, 580-592.

Bažant ZP and Oh BH (1985), Microplane model for progressive fracture of concrete and rock. J Eng Mech, ASCE, 111, 559-582.

Bažant ZP and Oh BH (1986), Efficient numerical integration on the surface of a sphere. Zeitschrift fur Angewandte Mathematik und Mechanik (ZAMM, Berlin), 66(1),37-49.

Bažant ZP and Prat P (1987), Creep of anisotropic clay: new microplane model. ASCE J Eng Mech, 103(7), 1050-1064.

Bažant ZP and Prat PC (1988a), Microplane model for brittle plastic materials: 1 . Theory. J Eng Mech, 114(10), 1672-1688.

Bažant ZP and Prat PC (1988b), Microplane model for brittle plastic materials: II. Verification. J Eng Mech, ASCE, 114, 1689-1702.

Bažant ZP, Xiang Y, and Prat PC (1996), Microplane model for concrete. I. Stress-strain boundaries and finite strain. J Eng Mech, ASCE, 122(3), 245-254, with Errata, Vol 123

Bell JF (1985), Contemporary perspectives in finite strain plasticity. Int $J$ Plasticity, 1, 3-27.

Berveiller M and Zaoui A (1978), An extension of the self-consistent scheme to plastically flowing polycrystals. JMech Phys Solids, 26, 325344.

Bishop JFW and Hill R (1951), A theory of the plastic distortion of a polycrystalline aggregate under combined stress. Phil Mag, 42, 414-427.

Brocca M, Brinson LC and Bažant ZP (1999a), Three dimensional constitutive model for shape memory alloys based on the microplane model. Submitted to J Mech Phys Solids.

Brocca M, Bažant ZP and Daniel IM (1999), Microplane model for stiff foams applied to the finite element analysis of failure by core indentation of sandwich beams. Submitted to ASME JMat Tech.

Bronkhorst CA, Kalindindi SR, and Anand L (1992), Polycristalline plasticity and the evolution od crystallographic texture in FCC metals. Phil Trans $R$ Soc London, A341, 443-477.

Brown GM (1970), A self-consistent polycrystalline model for creep under combined stress states. J Mech Phys Solids, 18, 367-382.

Brown SB, Kim KH, and Anand L (1989), An internal variable constitutive model for hot working of metals. Int J Plasticity.

Budiansky B and Wu TT (1962), Theoretical prediction of plastic strains in polycrystals. Proc of $4^{\text {th }}$ US Natl Congr Appl Mech, 1175-1 185.

Butler GC and McDowell DL (1998), Polycrystal constraint and grain subdivision. Int J Plasticity, 14(8), 703-717.

Caner FC and Bažant ZP (2000), Microplane model M4 for concrete: IÍ. Algorithm, calibration and application. J Eng Mech, ASCE 126(9) 954-961.

Canova GR, Kocks UF, and Jonas JJ (1984), Theory of torsion texture development. Acta Metall 32, 211.

Carol I and Bažant ZP (1993), Viscoelasticity with aging caused by solidification of nonaging constituent. JEng Mech, ASCE, 119(11), 2252-2269.

Carol I. and Bažant ZP (1997), Damage and Plasticity in Microplane Theory. Int $J$ Solids Struct 34(29), 3807-3835.

Carol I, Prat PC, and Bažant ZP (1992), New explicit microplane model for concrete: Theoretical aspects and numerical implementation. Int $J$ Solids Struct, 29(9), 1173-1191.

Christoffersen J and Hutchinson JW (1979), A class of phenomenological comer theories of plasticity. $J$ Mech Phys Solids, 27, 465-487.

Dienes JK (1979), On the analysis of rotation and stress rate in deforming bodies. Acta Mech, 32, 217-232.

Doyle TC and Ericksen JL (1956), Non-linear elasticity. Adv Appl Mech 4, 53-115. 
Drucker DC (1951), A More Fundamental Approach to Plastic Stress-Strain Relations. Proc of Ist US Natl Congress of Appl Mech, ASME, 487-491.

Eterovic AL and Bathe $\mathrm{KJ}$ (1990), A hyperelastic-based large-strain elastoplastic constitutive formulation. Int J Num Meth Eng, 30, 1099-11 14.

Flory TJ (1961), Thermodynamic relations for high elastic materials. Trans, Faraday Soc 57, 829-838.

Fressengeas $C$ and Molinari A (1985), Sur les critères de localisation de la déformation plastique de traction simple. $J$ Phys Paris Colloq 46(8), 121-125.

Gabriel G and Bathe KJ (1995), Some computational issues in large strain elasto-plastic analysis. Comput Struct, 56 (2/3), 249-267.

Gerard G and Becker H (1957), Handbook of structural stability: Part I Buckling of flat plates. NACA Tech Note 3781 .

Harren S, Lowe TC, Asaro RJ, and Needleman A (1989), Analysis of largestrain shear in rate-dependent face-centered cubic polycrystals: correlations of micro- and macromechanics. Phil Trans $R$ Soc Lond, A328, 443-500.

Hibbitt HD, Karlsson BI, and Sorensen P (1994), ABAQUS Theory Manual, Version 5.5 Hibbitt, Karlson and Sorensen Inc, Pawtucket, RI, Sec 4.6.1.

Hibbitt HD, Marcal PV, and Rice JR (1970), A finite element formulation for problems of large strain and large displacement. Int J Solids Struct, 6, $1069-1086$

Hill R (1950), The Mathematical Theory of Plasticity. Oxford Univ Press, Oxford.

Hill R (1958), A general theory of uniqueness and stability in elastic-plastic solids. J Mech Phys Solids, 6, 236-249.

Hill R (1959), Some basic principles in the mechanics of solids without a natural time. JMech Phys Solids, 7, 209-225.

Hill R (1965), Continuum micromechanics of elastoplastic polycrystals. $J$ Mech Phys Solids, 13, 89-102.

Hill R (1966), Generalized constitutive relations for incremental deformation of metal crystals by multislip. J Mech Phys Solids, 14, 95-102.

Hill R (1972), On constitutive macro-variables for heterogeneous solids at finite strain. Proc R Soc Lond, A326, 131-147.

Hill R and Rice JR (1972), Constitutive analysis of elastic-plastic crystal at arbitrary strain. JMech Phys Solids, 20, 401-413.

Hoger A (1987), The stress conjugate to logarithmic strain. Int $J$ Solids Struct, 23(12), 1645-1656.

Honneff $\mathrm{H}$ and Mecking $\mathrm{H}$ (1978), A method for the determination of the active slip systems and orientation changes during single crystal deformation. Proc of $5^{\text {th }}$ Int Conf On Texture of Materials (IOCOTOM 5), G Gottstein and K Lucke (eds), 1, 265-275.

Hughes DA (1995), The evolution of deformation microstructure and local orientation. Proc $16^{\text {th }}$ Riso Int Symp On Materials Science: Microstructural and Crystallographic Aspects of Recrystallozation, N Hansen, Y-L Liu, D Juul Jensen, and B Ralph (eds), 63-85. Riso Natl Lab, Roskilde, Denmark.

Hughes DA and Hansen N (1993), High Angle Boundaries and Orientation Distribution at large strains. Scripta Metall Mater, 33(2),315-321.

Hughes TJR (1984), Numerical implementation of constitutive models: rateindependent deviatoric plasticity. Theoretical Foundation for LargeScale Computations for Nonlinear Material Behavior, S Nemat-Nasser RJ Asaro, and GA Hegemier (eds), Matrinus Nijhoff Publ, Dordrecht, Netherlands.

Hutchinson JW (1965), Bounds and self-consistent estimates for creep of polycrystalline materials. Proc $R$ Soc London, A348, 101-127.

Hutchinson JW (1970), Elastic plastic behavior of polycrystalline metals and composites. Proc R Soc London, A319, 247-272.

Hutchinson JW (1974), Plastic buckling. Adv Appl Mech, 14, 67-144.

Hutchinson JW and Tvergaard V (1980), Surface instabilities in statically constrained plastic solids. Int J Mech Sci, 22, 339-354.

Johnson GR, et al. (1995), User instructions for the 1995 version of the EPIC Reaserch Code. Alliant Techsystems, Hopkins, New Mexico.

Key SW (1984), In Mechanics of elastic and inelastic solids 6: theoretical foundations for large-scale computations for nonlinear material behavior, S Nemat-Nasser, RJ Asaro, and GA Hegemier (eds). Dordrecht: Martinus Nijhoff Publ

Koiter WT (1953), Stress-strain relations, uniqueness and variational theorems for elastic-plastic materials with a singular yield surface. $Q$ Appl Math, 11, 350-354.

Krieg RD and Key SW (1976), Implementation of a time independent plasticity theory into structural computer programs. Constitutive Equations in Viscoplasticity: Computational and Engineering Aspects, JA Stricklin and KJ Saczlski (eds), AMD 20, ASME, New York, 125-137.

Kröner E (1961), Zur Plastischen Verformung des Vielkristalls . Acta Metall, 9, 155-161.

Lee EH, Mallett RL, and Wertheimer TB (1983), Stress analysis for anisotropic hardening in finite-deformation plasticity. J Appl Mech. Trans ASME, 50, 554-560.
Lin TH and Ito M (1965), Theoretical plastic distortion of a polycrystalline aggregate under combined and reversed stresses. $J$ Mech Phys Solids, $13,103-115$.

Lubliner J (1986), Normality rules in large-deformation plasticity. Mech Mat, 5, 29-34.

Malvern LE (1969), Introduction to Mechanics of a Continuous Medium, Prentice Hall, Englewood Cliffs NJ.

Mandel J (1965), Generalisation de la théorie de plasticité de WT Koiter. Int $J$ Solids Struct, 1, 273-295.

McMeeking RM and Rice JR (1975), Finite element formulation for problems of large elastic plastic deformation. Int J Solids Struct, 11, 601-616.

Needleman A (1972), A numerical study of necking in circular cylindrical bars. JMech Phys Solids, 20, 111-127.

Needleman A (1984), On finite element formulations for large elastic-plastic deformations. Comput Struct 20(1-3), 247-257.

Ogden RW (1984), Non Linear Elastic Deformations. Ellis Horwood, Ltd, Chichester UK and John Wiley and Sons, Chichester UK.

Osias JR and Swedlow JL (1974), Finite elasto-plastic deformation- I. theory and numerical examples. Int $J$ Solids Struct, 10, 321-339.

Pan J and Rice JR (1983), Rate sensitivity of plastic flow and implications for yield surface vertices. Int $J$ Solids Struct, 19, 973-987.

Pande G and Sharma K (1983), Multilaminate model of clays-A numerical evaluation of the influence of rotation of principal axes. $J$ Eng Mech, ASCE, 109(7), 397-418.

Pierce D, Asaro RJ, and Needleman A (1983), Material rate dependence and localized deformation in crystalline solids. Acta Metall, 31, 1951.

Ponthot J-P (1995), Radial retum extensions for visco-plasticity and lubricated friction. SmiRT-13 Int Conf on Structural Mechanics in Reactor Technology, Porto Alegre, Brazil.

Prat P and Bažant ZP (1989), Microplane model for triaxial deformation of soils. In Numerical Models in Geomechanics (NUMOG III), S Pietruszczac and G Pande (eds), 139-146. Elsevier, Niagara Falls, Canada.

Prat P and Bažant ZP (1991a), Microplane model for triaxial deformation of saturated cohesive soils. ASCE J Geotech Eng 117(6), 891-912.

Prat P and Bažant ZP (1991b), A time dependent microplane model for creep of cohesive soils. In Mechanics Computing in the 90s and beyond, Proc of ASCE-EMD Specialty Conf, H Adeli and RL Sierakowski (eds), 1224-1228, ASCE, Columbus OH

Rice JR (1993), Mechanics of solids. Encyclopedia Britannica, $15^{\text {th }}$ Edition, 23, 737-747 and 773 .

Rice JR (1971), Inelastic constitutive relations for solids: An internal variable theory and its application to metal plasticity. $J$ Mech Phys Solids, $19,433-455$.

Rollett AD, Stout MG, and Kocks UF (1989), Polycrystal plasticity as applied to the problem of in-plane anisotropy in rolled cubic metals. Proc of Plasticity '89, $2^{\text {nd }}$ Int Symp on Plasticity and its Current Applications, AS Kahn and M Tokuda (eds), Mie, 69-72, Japan.

Rudnicky JW and Rice JR (1975), Conditions for the localization of deformation in pressure-sensitive dilatant materials. $J$ Mech Phys Solids, 23, 371-394.

Sewell MJ (1974), Plastic flow rule at a yield vertex. J Mech Phys Solids, 22, 469-490

Sidoroff F (1974), Un modèle viscoélastique non linéaire avec configuration intermédiaire. Journal de Mécanique 13, 679-713.

Simo JC (1988), A framework for finite strain elastoplasticity based on maximum plastic dissipation and the multiplicative decomposition. Comput Meth Appl Mech Eng, 66, 199-219 and 68, 1-31.

Simo JC and Ortiz M (1985), A unified approach to finite deformation elasto-plasticity based on the use of hyperelastic constitutive equations. Comput Meth Appl Mech Eng 49, 177-208.

Stören S and Rice JR (1975), Localized necking in this sheets. J Mech Phys Solids, 23, 421-441.

Stowell EZ (1948a), Critical shear stress of an infinitely long plate in the plastic region. NACA Tech Note.

Stowell EZ (1948b), A unified theory of plastic buckling of columns and plates. $N A C A$, Rep. 898.

Stroud AH (1971), Approximate calculation of multiple integrals. PrenticeHall, Englewood Cliffs NJ.

Taylor GI (1934), The mechanics of plastic deformation of crystals. part itheoretical . Proc R Soc London, 145, 362-387.

Taylor GI (1938), Plastic Strain in Metals. J Inst Met, 63, 307-324.

Taylor GI and Elam CF (1923), The distortion of an aluminium crystal during a tensile test . Proc R Soc London, A102, 643-667.

Taylor GI and Elam CF (1925), The plastic extension and fracture of aluminium crystals. Proc R Soc London, 108, 28-51.

Taylor GI and Elam CF (1926), The distortion of iron crystals. Proc $R$ Soc London, 112, 337-361.

Valanis K (1971), A theory of viscoplasticity without a yield surface. $A r-$ chiwum Mechaniki Stossowanej (Archives of Mechanics, Warsaw) 23, $517-551$ 
Weber $G$ and Anand L (1990), Finite deformation constitutive equations and a time integration procedure for isotropic, hyperelastic-viscoplastic solids. Comp Meth appl Mech Engrg, 79, 173.

Wilkins ML (1964), Calculation of Elastic-plastic Flow. Methods of Conputational Physics, 3, B Alder et al (eds), Academic Press, New York.
Zienkiewicz $O$ and Pande G (1977), Time-dependent multi-laminate model of rocks- A numerical study of deformation and failure of rock masses. Ini J Anal Num Meth Geomech, 1, 219-247.

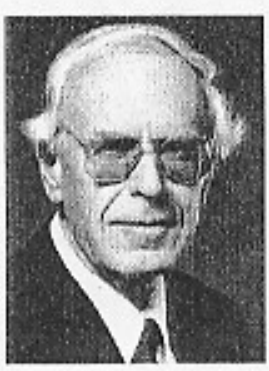

Zdenčk P Bažant, born and educated in Prague, joined Northwestern University faculty in 1969, became Professor in 1973, was named to the distinguished WP Murphy Chair in 1990, and served during 19811987 as Director of the Center for Concrete and Geomaterials (a predecessor to the ACBM Center). In 1996, he was elected to the National Academy of Engineering. He has authored 380 refereed journal articles and published books on Fracture and Size Effect (1997), Concrete at High Temperature (1996), Stability of Structures (1991), and Creep of Concrete (1966). He served as Editor (in chief) of the ASCE Journal of Engineering Mechanics (1988-1994) and is Regional Editor of the International Journal of Fracture. He was founding President of LA-FraMCoS, President of the Society of Engineering Science (SES), and Chairman of LA-SMiRT Division H. He is an Illinois Registered Structural Engineer, and chaired various committees in ASCE, RLEEM, and ACI. His honors incude: Prager Medal from SES, Warner Medal from ASME; Newmark Medal, Croes Medal, Huber Prize, and TY Lin Award from ASCE; L'Hermite Medal from RLEM; Torroja Gold Medal (Madrid); Humboldt Award; Honorary Doctorates from University of Colorado, CVUT Prague, and Universität Karlsruhe, Germany; Guggenheim, NATO, JSPS, and Kajima Fellowships; etc. He is a Foreign Member of the Austrian Academy of Sciences and Academy of Engineering of the Czech Republic, and a Fellow of the American Academy of Mechanics, ASME, ASCE, SES, ACI, and RILEM.

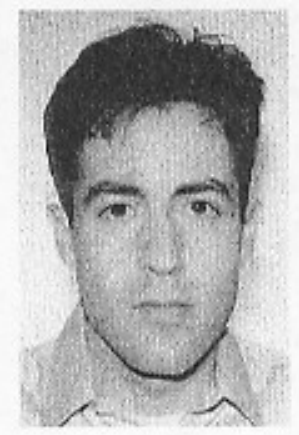

Michele Brocca earned his PhD in Civil Engineering from Northwestern University, Evanston, IL. He has been Research Assistant at the University of Tokyo and at Northwestern University and PostDoctoral Fellow at Northwestern University. 